Research Article

\title{
Inverse Approach to Evaluate the Tubular Material Parameters Using the Bulging Test
}

\author{
Yulong Ge, Xiaoxing Li, and Lihui Lang \\ School of Mechanical Engineering \& Automation, Beihang University, Beijing 100191, China \\ Correspondence should be addressed to Yulong Ge; aaronge@buaa.edu.cn
}

Received 12 October 2015; Revised 20 November 2015; Accepted 22 November 2015

Academic Editor: Pavel Lejcek

Copyright ( 2015 Yulong Ge et al. This is an open access article distributed under the Creative Commons Attribution License, which permits unrestricted use, distribution, and reproduction in any medium, provided the original work is properly cited.

\begin{abstract}
Tubular material parameters are required for both part manufactory process planning and finite element simulations. The bulging test is one of the most credible ways to detect the property parameters for tubular material. The inverse approach provides more effective access to the accurate material evaluation than with direct identifications. In this paper, a newly designed set of bulging test tools is introduced. An inverse procedure is adopted to determine the tubular material properties in KrupkowskiSwift constitutive model of material deformation using a hybrid algorithm that combines the differential evolution and LevenbergMarquardt algorithms. The constitutive model's parameters obtained from the conventional and inverse methods are compared, and this comparison shows that the inverse approach is able to offer more information with higher reliability and can simplify the test equipment.
\end{abstract}

\section{Introduction}

Tube hydroforming is one of the exclusive methods for the production of complex lightweight structures. This method is now widely used for manufacturing hollow parts in the automotive, aerospace, and civil industries. In comparison with stamping and welding, tube hydroforming has several advantages, such as weight reduction, improved structural properties, and reduced spring-back [1].

The success of tube hydroforming process design is highly dependent on accurate material properties for numerical simulations and the measurement of tubular material characteristics has always been a difficult issue in engineering. The required characteristics of the raw materials for quality hydroforming applications are a high strain hardening index and a low anisotropy [2].

Conventionally, approximate results are acquired by the sheet test. Wang et al. [3] developed the ring hoop tension test for transverse tensile properties of tubular materials. Fuchizawa and Narazaki [4] suggested that the tube was stretched in a biaxial fashion, which means that uniaxial material definition might lead to inaccuracy, and they introduced the bulging test to evaluate the material properties. Strano and Altan [5] described an approach to determine the flow stress of the tube parameters using the bulge test based on an energy balance perspective. This proposed technique is very straightforward and, therefore, suitable to be used on any hydroforming process. Kuwabara and Sugawara [6] developed a servocontrolled multiaxial tube expansion testing machine with a novel strain measurement apparatus for tubular specimens to measure the multiaxial plastic deformation behavior of tubular metals for a range of strains from initial yield to fracture. Koç et al. [7] explained the measurement of material properties of tubes under hydraulic bulging conditions and tooling design. Hwang et al. [8] used annealed AA6011 aluminum and SUS409 stainless steel tubes and a newly proposed analytical model to evaluate the tubular materials properties by hydraulic bulging. Thickness at the pole, bulging height, and the internal pressure were measured during test.

Because there are several disadvantages in the above studies, the inverse approach is introduced to determine the tubular material constitutive parameters. It is based on finite element (FE) simulations coupled with an optimization method and a set of experimental tests. This method allows for a more accurate determination of material parameters in comparison with direct identification. Gelin and Ghouati [9] applied this method to identify the viscous-plastic parameters 


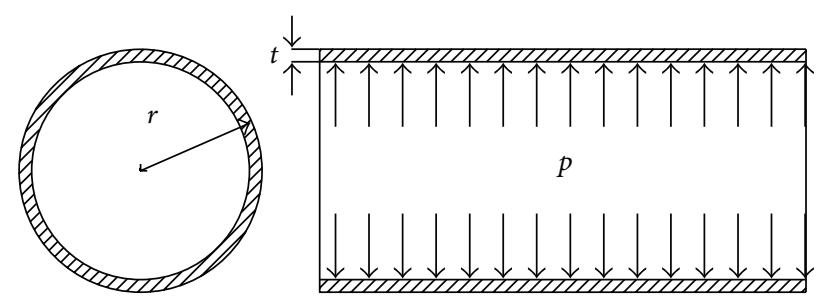

FIgURE 1: Tube blank under internal pressure.

that described the flow stress of an aluminum alloy for a large range of strains, strain rates, and temperatures. Anantharam [10] used the inverse approach to obtain the elastoplastic behavior of high speed steel (HSS) tools using the imprint profile of a Rockwell indentation. Zhou et al. [11] applied the inverse method to identify the constitutive parameters of an Al2O3sf/LY12 composite as well as other parameters. An inverse analysis methodology to simultaneously identify the parameters of various anisotropic yield criteria together with isotropic work-hardening models of metal sheets is outlined by Prates et al. [12].

Zribi et al. [13] used the inverse approach to test low carbon steel S235 and aluminum AA6063-O alloys. They compared their results to experimental data and concluded that the flow stress relationship using the inverse approach could be used to predict accurate plastic deformation behavior during the tube hydroforming process [14].

Of the above studies, few considered the influence of the elastic properties or the anisotropic coefficient.

\section{Analysis}

2.1. Tube Bulging Process. During the tube bulging process, the part blank can be seen as a thin wall spheroidal body that is revolved along its axis. When internal pressure $p$ is small, the blank is in the elastic state, in which the stresses can be written using the Lame formula as follows:

$$
\begin{aligned}
\sigma_{\theta} & =-\frac{2 p r^{2}}{t^{2}}, \\
\sigma_{z} & =0, \\
\sigma_{n} & =0,
\end{aligned}
$$

where $\sigma_{\theta}, \sigma_{z}$, and $\sigma_{n}$ are hoop stress, axial stress, and normal stress on the tubular thin wall, respectively. And $r$ is the initial outer diameter and $t$ is the initial thickness, respectively, as shown in Figure 1.

Regardless of the axial forces on the ends, the axial strain in the elastic state $\varepsilon_{z}$ can be calculated as follows:

$$
\varepsilon_{z}=-\frac{2 v r^{2}}{E t^{2}}
$$

where $E$ is Young's modulus and $v$ is Poisson's ratio. This equation indicates that the blank will deform along the tubular axis during the process.

During the internal pressure increase, the element on the middle section of the blank (pole element) rises, and the blank enters the plastic deformation state. The stressstrain state can be seen in Figure 2.

Considering the thin wall assumption, the normal stress on the elements can be ignored for simplicity. The balanced equation perpendicular to the axis on the pole element is as follows:

$$
\frac{\sigma_{z}}{\rho_{z}}+\frac{\sigma_{\theta}}{\rho_{\theta}}=\frac{p}{t}
$$

where $\rho_{z}, \rho_{\theta}$ are the axial and hoop curvature radiuses.

The axial forces imposed on both tube-ends and the bending moments are disregarded due to the assumption of a thin wall, and the equilibrium equation along the tube axis can be obtained as follows:

$$
2 \pi \rho_{\theta} t \sigma_{\phi}=\pi\left(\rho_{\theta}-t\right)^{2} p .
$$

Therefore, the axial stress $\sigma_{z}$ and the hoop stress $\sigma_{\theta}$ can be computed as follows:

$$
\begin{aligned}
\sigma_{z} & =\frac{p\left(\rho_{\theta}-t\right)^{2}}{2 t \rho_{\theta}}, \\
\sigma_{\theta} & =\rho_{\theta}\left(\frac{p}{t}-\frac{\sigma_{z}}{\rho_{z}}\right) .
\end{aligned}
$$

According to Hill's 48 yielding criteria, the effective stress $\bar{\sigma}$ and effective strain rate $d \bar{\varepsilon}$ can be written as follows:

$$
d \bar{\varepsilon}=\frac{1+r}{\sqrt{1+2 r}} \sqrt{d \varepsilon_{\theta}^{2}+\frac{2 r}{2+r} d \varepsilon_{\theta} d \varepsilon_{z}+d \varepsilon_{z}^{2}},
$$

where $r$ represents the anisotropic coefficient and $d \varepsilon_{\theta}$ and $d \varepsilon_{z}$ represent the hoop and axial rate, respectively. They are associated with the stress state and deformation history based on the plastic incremental theory. These relations can be expressed in plane stress state as

$$
\frac{d \varepsilon_{\theta}}{\sigma_{\theta}-(r /(1+r)) \sigma_{z}}=\frac{d \varepsilon_{z}}{\sigma_{z}-(r /(1+r)) \sigma_{\theta}}=\frac{d \bar{\varepsilon}}{\bar{\sigma}} .
$$

And the normal strain rate $d \varepsilon_{t}$ can be obtained using the incompressibility of plastic deformation as

$$
d \varepsilon_{t}=-d \varepsilon_{\theta}-d \varepsilon_{z} .
$$

2.2. Evaluation of Tubular Material Constitutive Model. The conventional methods to evaluate the constitutive model using bulging test are based on the following assumptions: (a) the strain path of the pole element is always linear; (b) the pole element proximity profile of the axial section is a cosine curve; (c) the materials obey the von Mises yield criterion $(r=1)$.

Therefore, the rates of strains can be replaced by the strains themselves. The effective stress and effective strain can be simplified in deflection theory as

$$
\frac{\varepsilon_{\theta}}{\sigma_{\theta}-(1 / 2) \sigma_{z}}=\frac{\varepsilon_{z}}{\sigma_{z}-(1 / 2) \sigma_{\theta}}=\frac{\bar{\varepsilon}}{\bar{\sigma}} .
$$




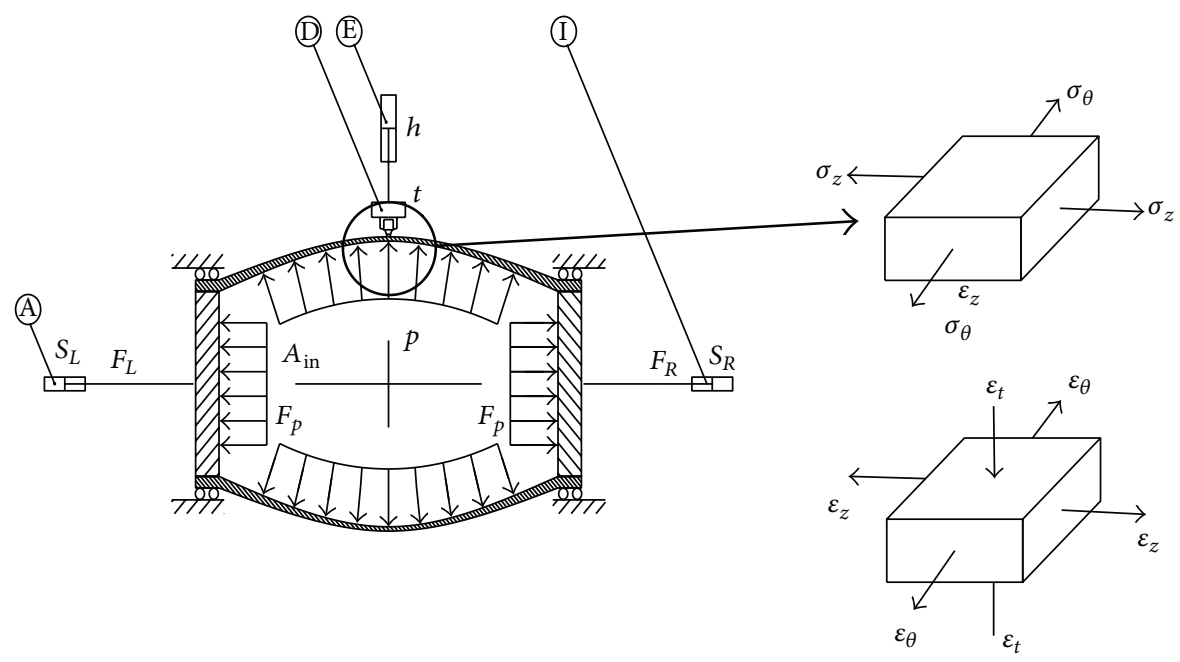

Figure 2: Stress-strain state on the pole element during the bulging test.

The hoop strain and axial strain can be computed using three displacement transducers as

$$
\begin{aligned}
& \varepsilon_{\theta}=\ln \frac{h_{1}}{h_{1,0}}, \\
& \varepsilon_{z}=\frac{\int_{-d}^{d} \sqrt{\left(\left(h_{1}-h_{2}\right)^{2} \pi^{2} / 4 d^{2}\right) \sin x+1} d x}{2 d},
\end{aligned}
$$

where the geometry relationship is shown in Figure 3 and the outer profile is assumed as a cosine curve.

For tubular materials, the constitutive models are always descripted in the Krupkowski-Swift hardening law. So the stress-strain state on the pole element can be expressed as

$$
\bar{\sigma}=K \times\left(\varepsilon_{0}+\bar{\varepsilon}\right)^{n},
$$

where $K$ is the strength coefficient, $\varepsilon_{0}$ is the prestrain, and $n$ is the harden index.

The methods like ring hoop tension test and traditional bulging test can draw a conclusion that using these methods is hard to access Young's module, Poison's ration, or anisotropic coefficient. Therefore, the inverse approach is introduced to evaluate the material parameters of tube. The principle of this method is to minimize the objective function that represents the error between the simulation result and the experimental result using an optimization algorithm, shown in Figure 4.

In the finite element method, the strain rate decomposition is $d \varepsilon=d \varepsilon^{\mathrm{el}}+d \varepsilon^{p}$, so the elastic parameters can be distinguished from plastic parameters. Meanwhile, Hill's 48 anisotropic plasticity potential is also defined. It means $E$, $v$, and $r$ can be taken into consideration using FEM. Therefore, inverse approach can determine the parameters that are not easily obtained in conventional ways. In this study, the objective function is defined as mean square error (MSE) function expressed as

$$
F_{\text {obj }}=\frac{\sum_{i=1}^{N}\left(h_{i}^{\mathrm{FEM}}-h_{i}^{\mathrm{exp}}\right)}{N},
$$

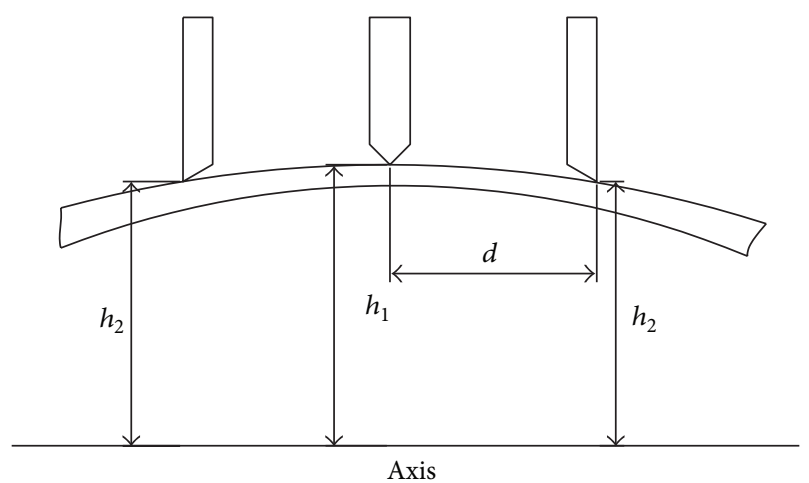

Figure 3: Geometry relation to compute strains.

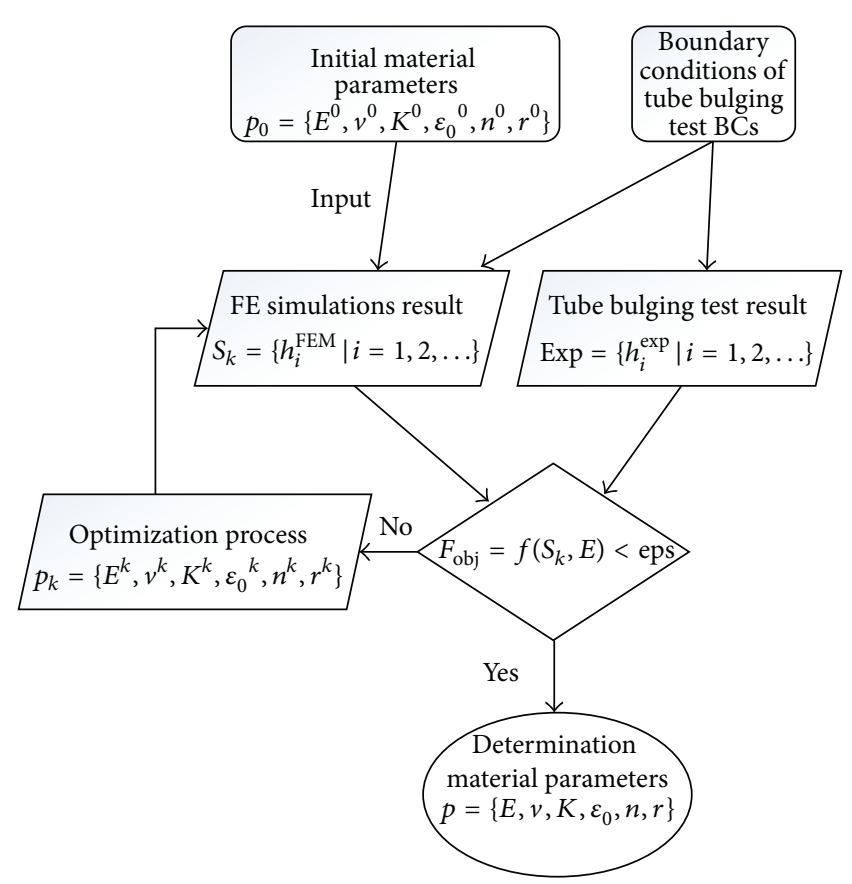

FIGURE 4: Inverse approach principle. 


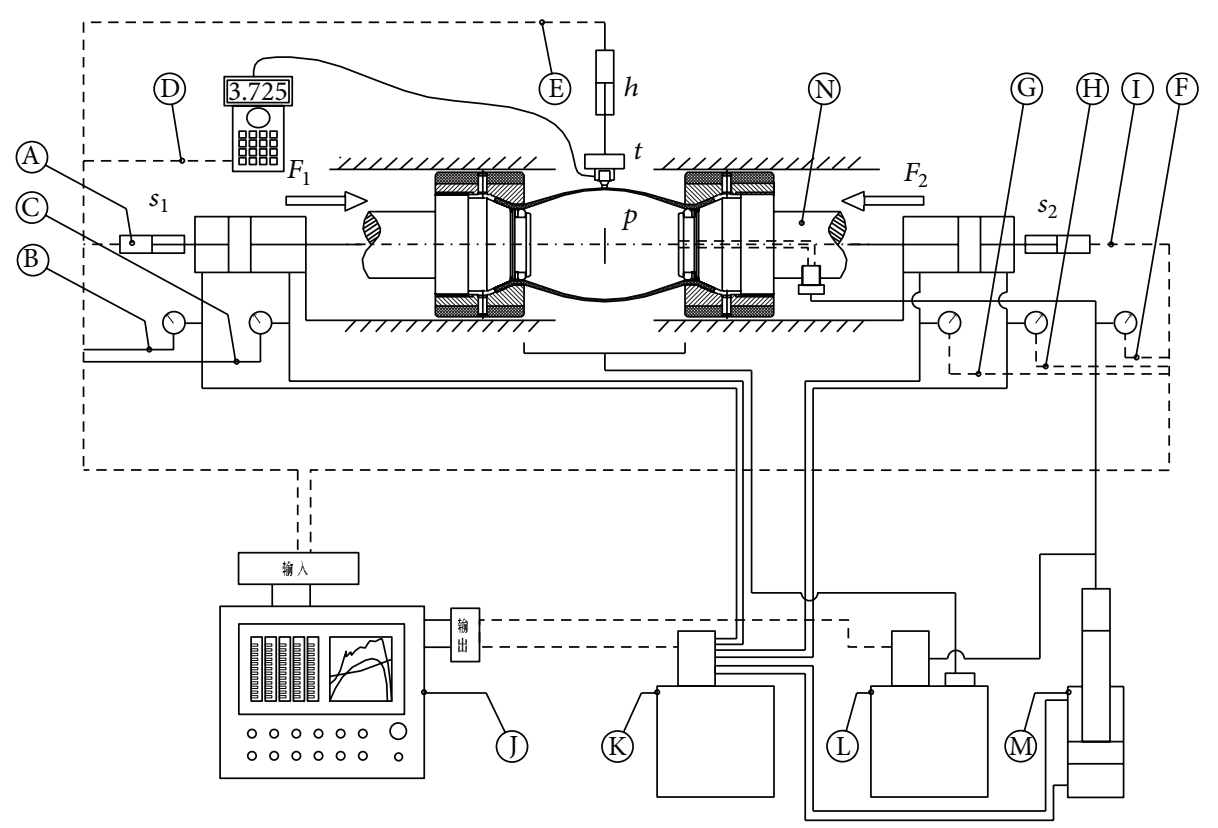

$\begin{array}{ll}\text { A: Displacement transducer } & \text { H: Pressure sensor } \\ \text { B: Pressure sensor } & \text { I: Displacement transducer } \\ \text { C: Pressure sensor } & \text { J: PLC } \\ \text { D: Audigage } & \text { K: Hydraulics } \\ \text { E: Displacement transducer } & \text { L: Forming water system } \\ \text { F: Pressure sensor } & \text { M: Booster } \\ \text { G: Pressure sensor } & \end{array}$

FIGURE 5: Tool set for bulging test.

where $h_{i}^{\mathrm{FEM}}$ and $h_{i}^{\text {exp }}$ are the simulation and experimental bulging height points under the same internal, respectively. $N$ is the number of measuring points. In this way, the objective function can indicate the whole deformation process of bulging test. Elastic parameters account for major rule of the deformation process at first, and plastic parameters raise their impacts during the pressure increasing.

As discussed above, the feasibility of the inverse approach mainly depends on the accuracy of the experimental data, proper finite element model, and a reliable algorithm.

\section{Implement Method}

3.1. Tool Set. As the foundation of experiments, this paper presents a newly designed tube bulging test machine with accurate boundary conditions and arbitrary axial force for the inverse approach, as shown in Figure 5. This machine consists of a servocontrol system, a hydraulic system, a water cycle system, a hydraulic intensifier, and tool sets, and it is capable of controlling the axial forces and internal pressure in the specimen. The system simultaneously monitors the realtime data from the displacement sensors for the left and right axial feeds as well as the bulging height at the pole point, the ultrahigh pressure sensor for the internal pressure $p$, and the pressure sensors for the rod and rodless cavities in the left and right cylinders. To access the free axial movement, the other degrees of freedom of the tube-end nodes must be well constrained. Coupled with the free-sliding boundary conditions, all of the axial forces provided by the lateral thrust cylinders will be applied accurately on the tube-ends. Due to the presence of flares and threaded fasteners, the stretch force can also be exerted on the tube-ends, and the clearance fit between the flaring tools and the guide rail is chosen to reduce the influence of friction [15].

A new control strategy named as "force-active and displacement-following" has been developed and validated after repeated experiments. The aim of the strategy is to drive the forces on the thrust cylinders $F_{1}$ and $F_{2}$ satisfying

$$
\begin{aligned}
& F_{1}=F_{2}=p A_{\text {in }}, \\
& s_{1}=s_{2},
\end{aligned}
$$

where $A_{\text {in }}$ is the initial inner cross-sectional area of the tube blank. $s_{1}$ and $s_{2}$ are the displacements of the left and right cylinders, respectively. To achieve the desired results, the left cylinder is force controlled while right cylinder is displacement controlled. The displacement signal from left is the input controlling signal of the right one in real-time, shown in Figure 6. Therefore, the bulging pole can be kept in the center. 


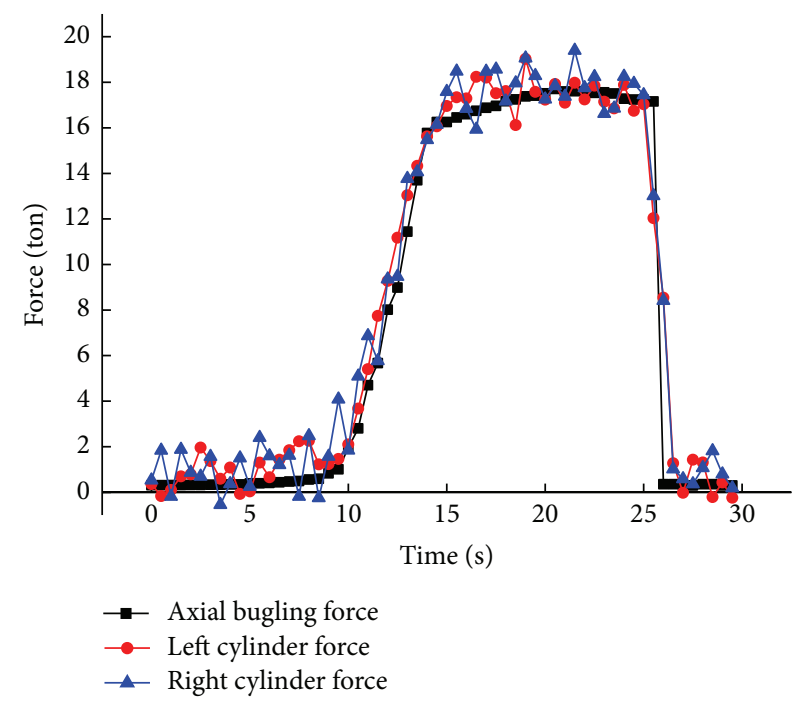

(a) Controlled forces

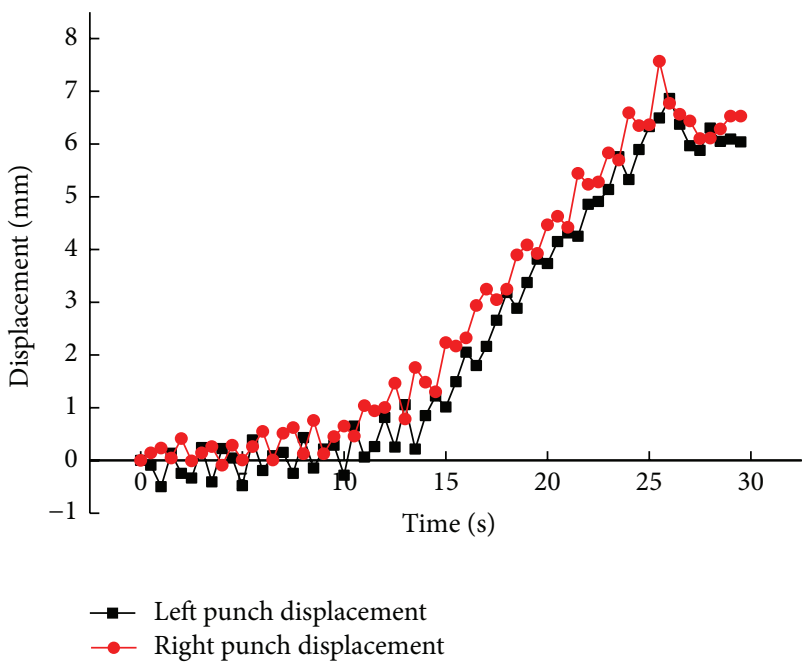

(b) Controlled displacements

FIGURE 6: Forces and displacements curve under controlling.

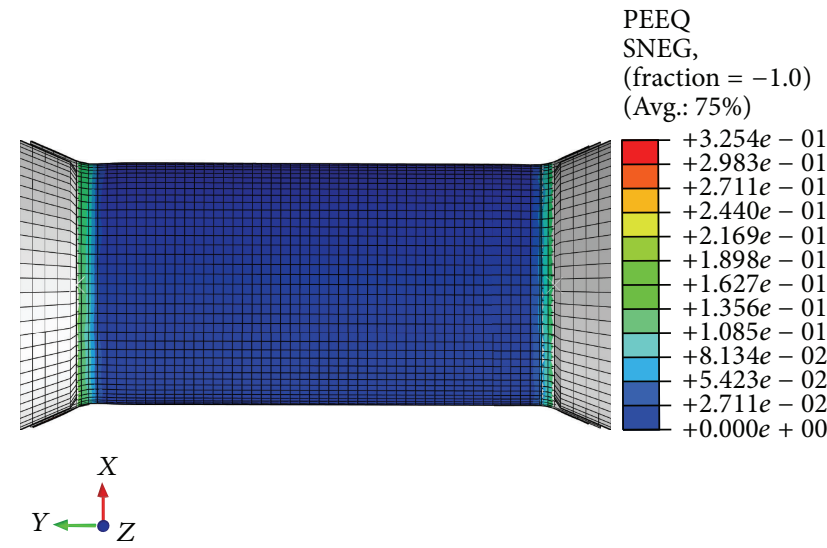

(a) Blank after flaring

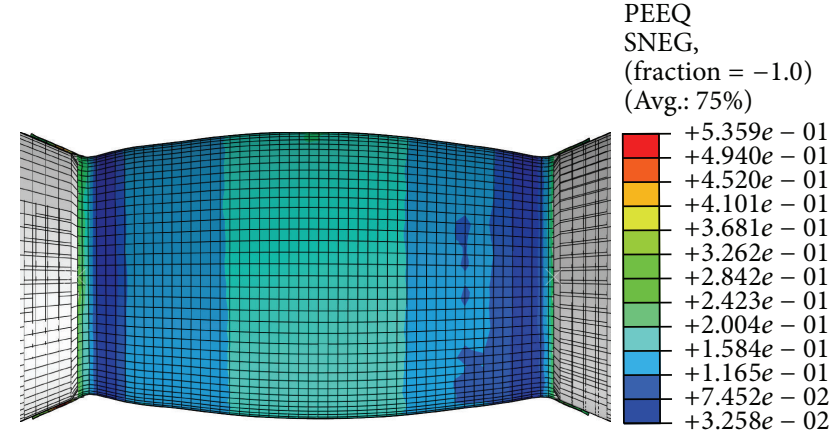

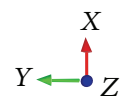

FIGURE 7: Finite element model for the inverse approach.

The tube-ends have been expanded by flaring molds before bulging test using the flaring angle of $23^{\circ}$ and the flaring depth of $20 \mathrm{~mm}$, so that the flanging can be clamped by the clamping nuts and pushing-rods through threaded fasteners. The material flow towards the centrally expanding region is held back; thus the length of deformation zone remains coincident. Additionally, the original length of tube bulging deformation region is also determined in this step.

3.2. Finite Element Model. In this study, ABAQUS/Explicit software is selected to accomplish this work because it contains easy access to the Python language [16]. A 3D elastoplastic FE model is built and shell S4R element is used for meshing the blank because it is a robust and general-purpose element that is suitable for a wide range of applications with uniformly reduced integration to avoid shear and membrane locking. Tool set is also meshed using
S4R and S3R elements and then tied to the reference points to be rigid bodies. The friction between blank and tools is set to 0.125 using penalty method. The simulation consists of two steps combined with restart technology: the flaring step and bulging step. In the flaring step, the molds move along the tubular axis to expand the tube-ends; in the bulging step, the molds are tied to the tube-ends so they can move with deformation. The pressure is imposed on the inner surface of blank and the boundary conditions are set axial free to imitate the experiment. The pressure versus time curve is designed just like the experimental one to eliminate the influence of loading path. Figure 7 shows the finite element simulation of bulging process.

3.3. Optimization Algorithm for Inverse Approach. NelderMead Simplex (NMS) algorithm is commonly adopted to obtain optimal result of objective function in the past works. 


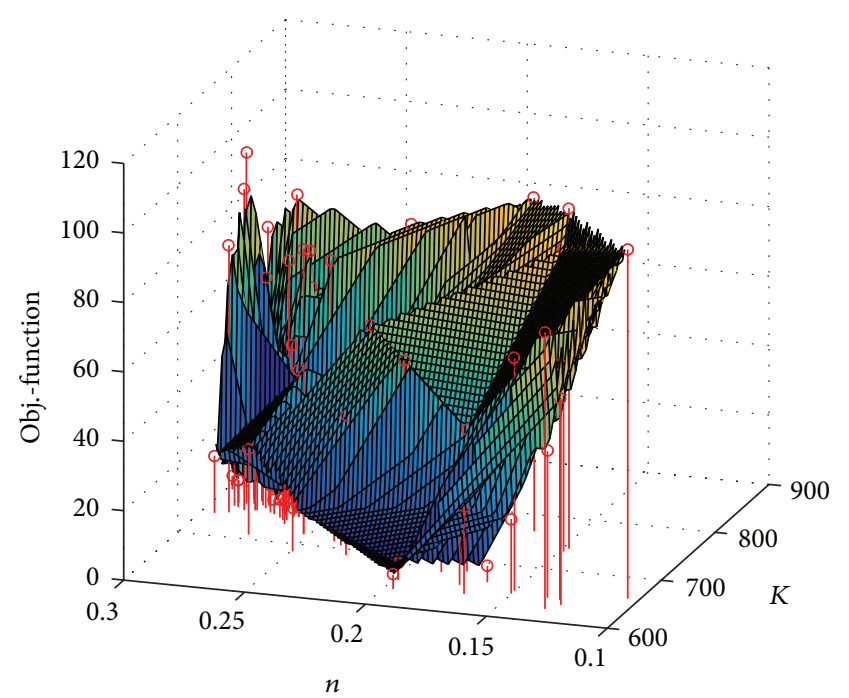

FIGURE 8: Local convergence points.

NMS algorithm has an obvious weakness that the convergence becomes increasingly difficult for problems with more than five design variables. Moreover, as the deformation process is a nonlinear system, there is a common problem that occurred in past works such that it is hard to avoid the entrapment in local minimum. Especially with high dimensions, premature convergence may occur and lead to a mistake result; see Figure 8.

To solve this problem, several random sets of initial parameters fallen in the range are used to get the minimums concurrently. Once the obtained results are similar in each case, it indicates that the global minimums are found. It could be very time-consuming in practice [13].

In this paper, a new hybrid optimization algorithm is adopted in this paper. Both differential evolution (DE) algorithm and Levenberg-Marquardt (LM) algorithm are applicable to this situation. The area wherein the global solution lies can be found using DE process and the LM process can search the local optimal solution acceleration.

Differential evolution is a stochastic population-based method for global optimization. Three main elementary operations in this algorithm are mutation, crossover, and selection. The crucial idea behind $\mathrm{DE}$ is a scheme for generating trial parameter vectors. Basically, DE generates new parameter vectors by adding the weighted difference between two population vectors to a third vector. If the resulting vector yields a lower objective function value than a predetermined population member, the newly generated vector replaces the previous vector to which it was compared in the next generation. Otherwise, the previous vector is retained [17]. Two parameters are adopted in DE: cross ratio $\mathrm{CR}$ and scaling factor $F$. Higher CR means less influence of the parent in the features of its offspring, which would lead to a decline in the capacity to handle complicated problems. $F$ controls the convergence of the process. Lower $F$ may cause an immature convergence. Otherwise, it may slow the speed. In this study, CR is chosen between 0 and 1.0 randomly and

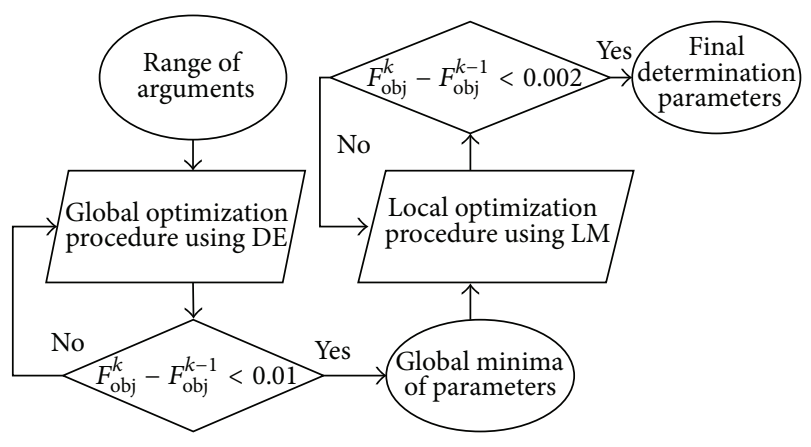

FIGURE 9: Optimization process of the inverse approach.

$F$ has the value 0.5 . Thus, the algorithm can search the global minima in an acceptable time [18].

The Levenberg-Marquardt (LM) algorithm is a gradientbased iterative technique that locates the minimum of a function that is expressed as the sum of the squares of nonlinear functions and can be thought of as a combination of the steepest descent and Gauss-Newton methods [19]. It is widely used as a standard technique for least-squares problems and this method has shown some nice local convergence properties under fairly mild assumptions [20]. Thus, it can be used to polish the best population member when DE is accomplished, which can improve the minimization slightly. In $\mathrm{FE}$ analysis, no explicit gradient matrixes can be acquired. To solve this problem, finite difference approximated Jacobian and Hessian matrixes are applied instead.

When combining these algorithms, the hybrid multistep algorithm has an important feather that it can guarantee the global optimal solution and local optimal solution.

The independent arguments are the six desired material parameters $E, v, K, \varepsilon_{0}, n$, and $r$. For each iteration, FE model using the current parameters is simulated. Waiting for the complement of the simulation, the designated characteristics are obtained from the results for objective function. Figure 9 shows the flow chart of the optimization process using hybrid algorithm.

\section{Experiments and Discussion}

In [14], the bulging height at pole dome versus the internal pressure is also used as the experimental data but the thinning process is used as validation. However, ultrasonic thickness probe cannot give precise thickness for a curved specimen, especially when the tube is filled with pressure medium. Therefore, it is not that convincing in this work. To avoid these problems, the tube-ends displacements history during the bulging process is used as the validation, which can be recorded in real-time by the sensors as discussed above.

To substantiate the effectiveness of this inverse approach to evaluate tubular material parameters, two stainless ERW steel tubular materials $\mathrm{H} 340$ and E355 are tested in this study. The original length of specimens is $210 \mathrm{~mm}$ (the length of clamping section at each end equal to $32.5 \mathrm{~mm}$ ). Thus, the deformation region was of $145 \mathrm{~mm}$ length. The ratio of length to outer diameter is 2.0 and the initial wall thickness is 
TABLE 1: Chemical composition (Wcr).

\begin{tabular}{lccccc}
\hline Designations & C & $\mathrm{Si}$ & $\mathrm{Mn}$ & $\mathrm{P}$ & $\mathrm{S}$ \\
\hline H340 & $\leq 0.1$ & $\leq 0.5$ & $\leq 1.0$ & $\leq 0.025$ & $\leq 0.025$ \\
E355 & $\leq 0.22$ & $\leq 0.55$ & $\leq 1.6$ & $\leq 0.025$ & $\leq 0.025$ \\
\hline
\end{tabular}

TABLE 2: Initial range of material parameters of H340 and E355.

\begin{tabular}{lcc}
\hline Parameter & H340 & E355 \\
\hline$E(\mathrm{GPa})$ & $170 \sim 250$ & $170 \sim 250$ \\
$v$ & $0.2 \sim 0.4$ & $0.2 \sim 0.4$ \\
$K(\mathrm{MPa})$ & $600 \sim 900$ & $800 \sim 1100$ \\
$\varepsilon_{0}$ & $0.001 \sim 0.01$ & $0.001 \sim 0.01$ \\
$n$ & $0.15 \sim 0.3$ & $0.1 \sim 0.25$ \\
$r$ & $0.5 \sim 3$ & $0.5 \sim 3$ \\
\hline
\end{tabular}

TABLE 3: Inverse approach evaluation results of H340 and E355.

\begin{tabular}{lcc}
\hline Parameter & H340 & E355 \\
\hline$E(\mathrm{GPa})$ & 196 & 204 \\
$\nu$ & 0.315 & 0.283 \\
$K(\mathrm{MPa})$ & 745.2 & 908.2 \\
$\varepsilon_{0}$ & 0.073 & 0.0336 \\
$n$ & 0.251 & 0.156 \\
$r$ & 1.27 & 1.13 \\
\hline
\end{tabular}

$3.75 \mathrm{~mm}$. The tubular blank is supplied by BAOSTEEL. The chemical compositions of the tubes can be seen in Table 1 .

For each material, three sets of experiments are implemented. The bulging height versus internal pressure curves in each test measured in real-time that are constituent parts of objective functions is shown in Figure 10. According to the plastic theory, if the stress passes the yielding point, a small increase of stress can engender a large strain. It can be reflected in bulging process. When the internal pressure reaches up to a certain extent, the bulging height will grow obviously under a little pressure increment. Although there are slight fluctuations, it can be found that the stability of bulging test machine is satisfactory. The experimental process of the bulging test is shown in Figure 11.

The initial trial range of the arguments is listed in Table 2; it depends on the height versus pressure curves trends of different tubular materials. From Figure 10, it can be deduced that the strength coefficient $K$ of E355 is higher than H340 and the hardening index $n$ is lower. It took about 16 hours and 14 hours to complete the computations with a quad core $2.5 \mathrm{GHz} \mathrm{CPU}$. The material parameters of $\mathrm{H} 340$ and E355 tubular materials obtained using the inverse approach evaluation can be seen in Table 3. And the flow stress curves are shown in Figure 12.

The flow stress curve of $\mathrm{H} 340$ tubular material using tensile test is also shown in Figure 12. The specimen for the tensile test is cut directly from the tube in the axial direction. The similar tendency can be found in work performed in [8].

In order to check the inverse approach evaluation ability to describe the bulging test behavior, FE simulations of bulging test are run using tubular material parameters from

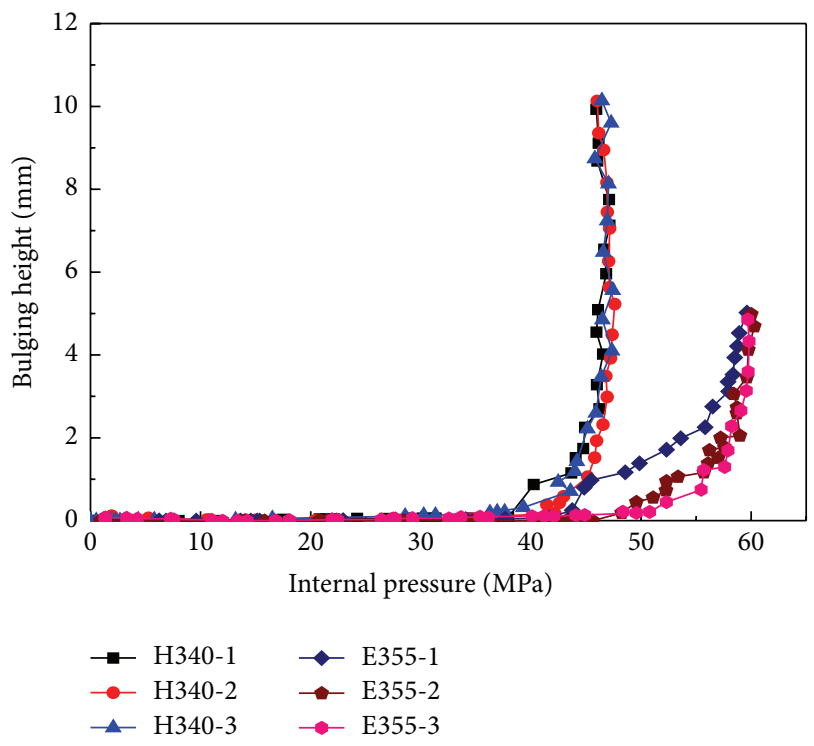

FIGURE 10: Bulging height versus internal pressure curves in experiments.

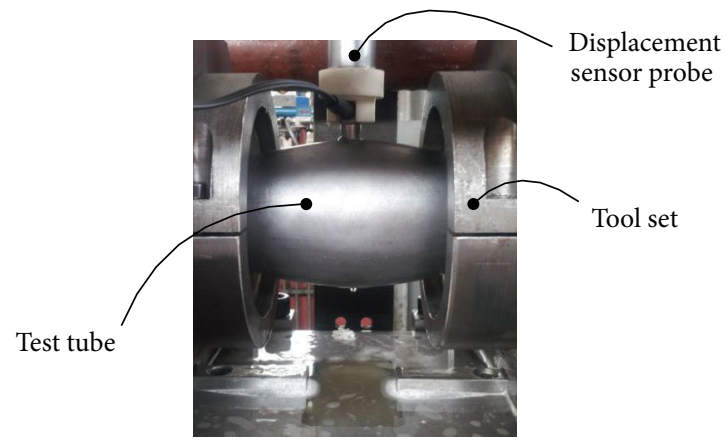

FIGURE 11: Process of tube bulging experiment.

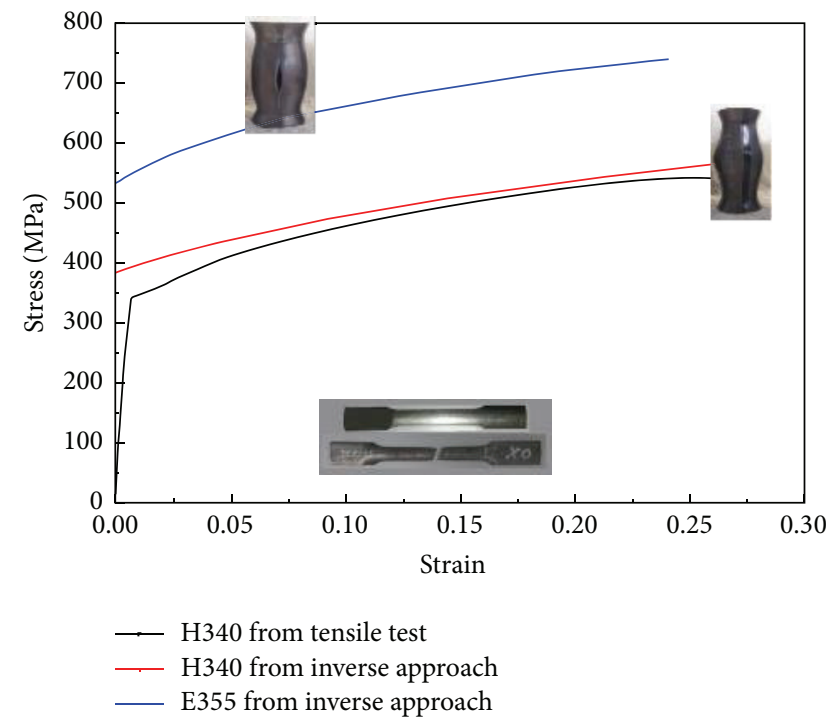

Figure 12: Stress-strain curves of H340 and E355. 


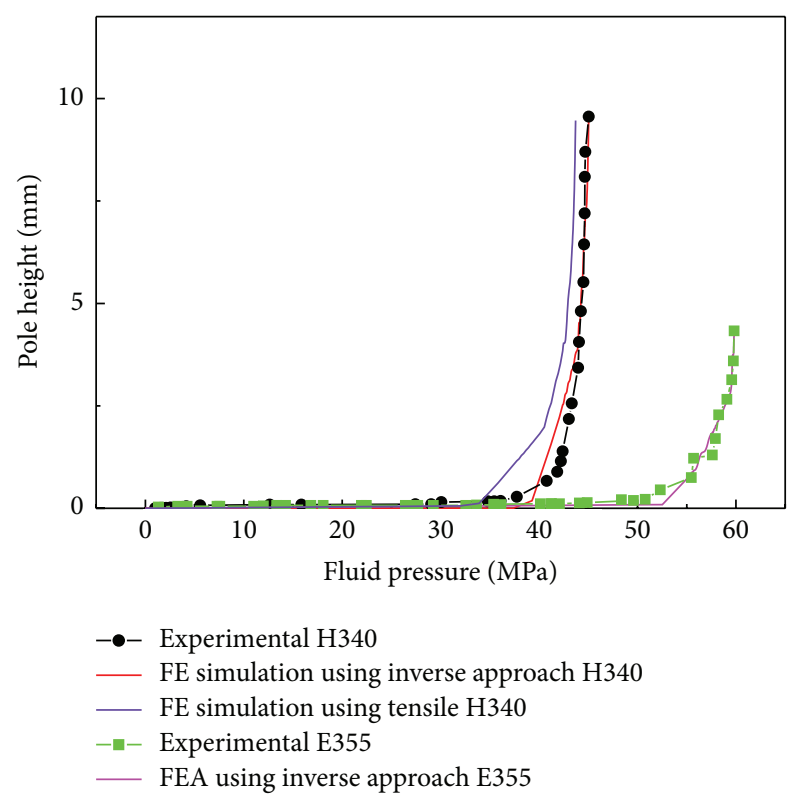

FIgURE 13: Comparison of the bulging height between the FE simulation and experimental results.

inverse approach and tensile test of $\mathrm{H} 340$ as inputs. Figure 13 shows the outputs (bulging height versus internal pressure) of these simulations and experimental ones. The inverse approach response is significantly narrower than tensile response. Output using E355 is also demonstrated and the gap from inverse approach response is quite small likewise. There is no doubt that the inverse approached parameters provide more accurate FE simulations of tube hydroforming.

To validate the prediction accuracy of inverse approach to tubular materials using bulging test, a task is conducted by comparing the experimental tube-ends displacement with the FE simulation ones. Referring to Figure 14, the tubeends displacement predicted using inverse approach of H340 is much better according to the experimental and the final profile by the inverse approach is in good agreement with the experimental values. The predicted ends displacement from FE simulation using tensile tested parameters is clearly in disagreement with the corresponding experimental results. E355 tubular bulging test can also be taken into consideration for further validation.

It can be concluded that the tubular material parameters obtained from inverse approach using bulging test are more adequate to describe the constitutive models and can lead to a more accurate FE simulation of tube hydroforming process.

\section{Conclusion}

In this study, an inverse approach is used to determinate the tubular material parameters using bulging test, including elastic parameters, flow stress parameters, and anisotropic coefficients. A hybrid algorithm combining differential evolution and Levenberg-Marquardt algorithms is used for the optimization in the inverse process. Experiments using a new designed bulging test machine for H340 and E355 tubular

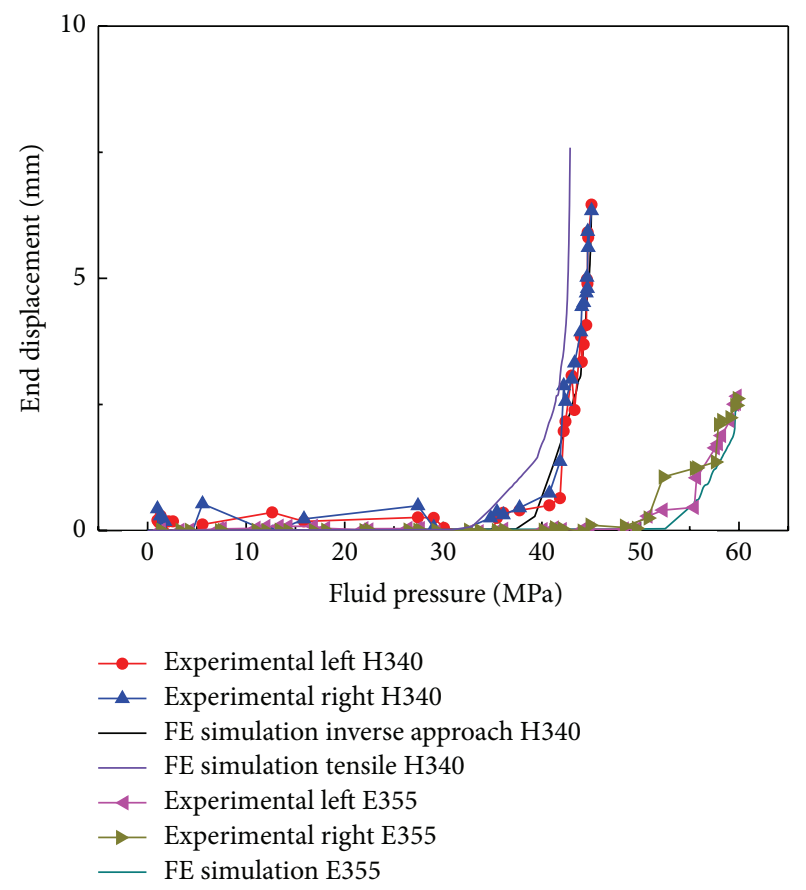

FIGURE 14: Comparison of the end displacement between the FE simulation and experimental results.

materials are carried out. The tensile test is also performed as object of comparing. It can be found that the material parameters inverse approach evaluated using bulge test can provide more accurate parameters to describe and predict the tube hydroforming tube behavior.

Moreover, the new designed bulging test machine for inverse approach requires three displacement sensors only for both evaluation and validation. Thus, it can simplify the equipment used for tubular bulging itests compared to the equipment in [9].

\section{Conflict of Interests}

The authors declare that there is no conflict of interests regarding the publication of this paper.

\section{References}

[1] M. Ahmetoglu and T. Altan, "Tube hydroforming: state-ofthe-art and future trends," Journal of Materials Processing Technology, vol. 98, no. 1, pp. 25-33, 2000.

[2] M. Ko, Hydroforming for Advanced Manufacturing, Elsevier, New York, NY, USA, 2008.

[3] H. Wang, R. Bouchard, R. Eagleson, P. Martin, and W. R. Tyson, "Ring hoop tension test (RHTT): a test for transverse tensile properties of tubular materials," Journal of Testing and Evaluation, vol. 30, no. 5, pp. 382-391, 2002.

[4] S. Fuchizawa and M. Narazaki, "Bulge test for determining stress-strain characteristics of thin tubes," in Advanced Technology of Plasticity, vol. 1, pp. 488-493, 1993.

[5] M. Strano and T. Altan, "An inverse energy approach to determine the flow stress of tubular materials for hydroforming 
applications," Journal of Materials Processing Technology, vol. 146, no. 1, pp. 92-96, 2004.

[6] T. Kuwabara and F. Sugawara, "Multiaxial tube expansion test method for measurement of sheet metal deformation behavior under biaxial tension for a large strain range," International Journal of Plasticity, vol. 45, pp. 103-118, 2013.

[7] M. Koç, Y. Aue-U-Lan, and T. Altan, "On the characteristics of tubular materials for hydroforming-experimentation and analysis," International Journal of Machine Tools and Manufacture, vol. 41, no. 5, pp. 761-772, 2001.

[8] Y.-M. Hwang, Y.-K. Lin, and T. Altan, "Evaluation of tubular materials by a hydraulic bulge test," International Journal of Machine Tools and Manufacture, vol. 47, no. 2, pp. 343-351, 2007.

[9] J. C. Gelin and O. Ghouati, "An inverse method for material parameters estimation in the inelastic range," Computational Mechanics, vol. 16, no. 3, pp. 143-150, 1995.

[10] B. S. Anantharam, Study of Break Down of Thin Hard Surface Coatings with Emphasis on FInite Element Simulations, Aalborg University, Aalborg, Denmark, 2004.

[11] J.-M. Zhou, L.-H. Qi, and G.-D. Chen, "New inverse method for identification of constitutive parameters," Transactions of Nonferrous Metals Society of China (English Edition), vol. 16, no. 1, pp. 148-152, 2006.

[12] P. A. Prates, M. C. Oliveira, and J. V. Fernandes, "Identification of material parameters for thin sheets from single biaxial tensile test using a sequential inverse identification strategy," International Journal of Material Forming, pp. 1-25, 2015.

[13] T. Zribi, A. Khalfallah, and H. Belhadjsalah, "Inverse method for flow stress parameters identification of tube bulge hydroforming considering anisotropy," International Journal of Mechatronics and Manufacturing Systems, vol. 4, no. 5, pp. 441453, 2011.

[14] T. Zribi, A. Khalfallah, and H. BelHadjSalah, "Experimental characterization and inverse constitutive parameters identification of tubular materials for tube hydroforming process," Materials \& Design, vol. 49, pp. 866-877, 2013.

[15] P. Z. Cheng, L. H. Lang, Y. L. Ge et al., "Tube free bulging experiment with force-end and material properties testing," Journal of Beijing University of Aeronautics and Astronautics, vol. 41, no. 4, pp. 686-692, 2015.

[16] F. Gassara, R. Hambli, T. Bouraoui, F. E. Halouani, and D. Soulat, "Optimization of springback in L-bending process using a coupled Abaqus/Python algorithm," International Journal of Advanced Manufacturing Technology, vol. 44, no. 1-2, pp. 61-67, 2009.

[17] R. Storn, "On the usage of differential evolution for function optimization," in Proceedings of the Biennial Conference of the North American Fuzzy Information Processing Society (NAFIPS '96), pp. 519-523, IEEE, Berkeley, Calif, USA, June 1996.

[18] E. Mezura-Montes, M. Reyes-Sierra, and C. A. Coello Coello, "Multi-objective optimization using differential evolution: a survey of the state-of-the-art," in Advances in Differential Evolution, U. K. Chakraborty, Ed., vol. 143 of Studies in Computational Intelligence, pp. 173-196, Springer, Berlin, Germany, 2008.

[19] M. I. A. Lourakis, A Brief Description of the LevenbergMarquardt Algorithm Implemented by Levmar, 2005.

[20] C. Kanzow, N. Yamashita, and M. Fukushima, "LevenbergMarquardt methods with strong local convergence properties for solving nonlinear equations with convex constraints," Journal of Computational and Applied Mathematics, vol. 172, no. 2, pp. 375-397, 2004. 

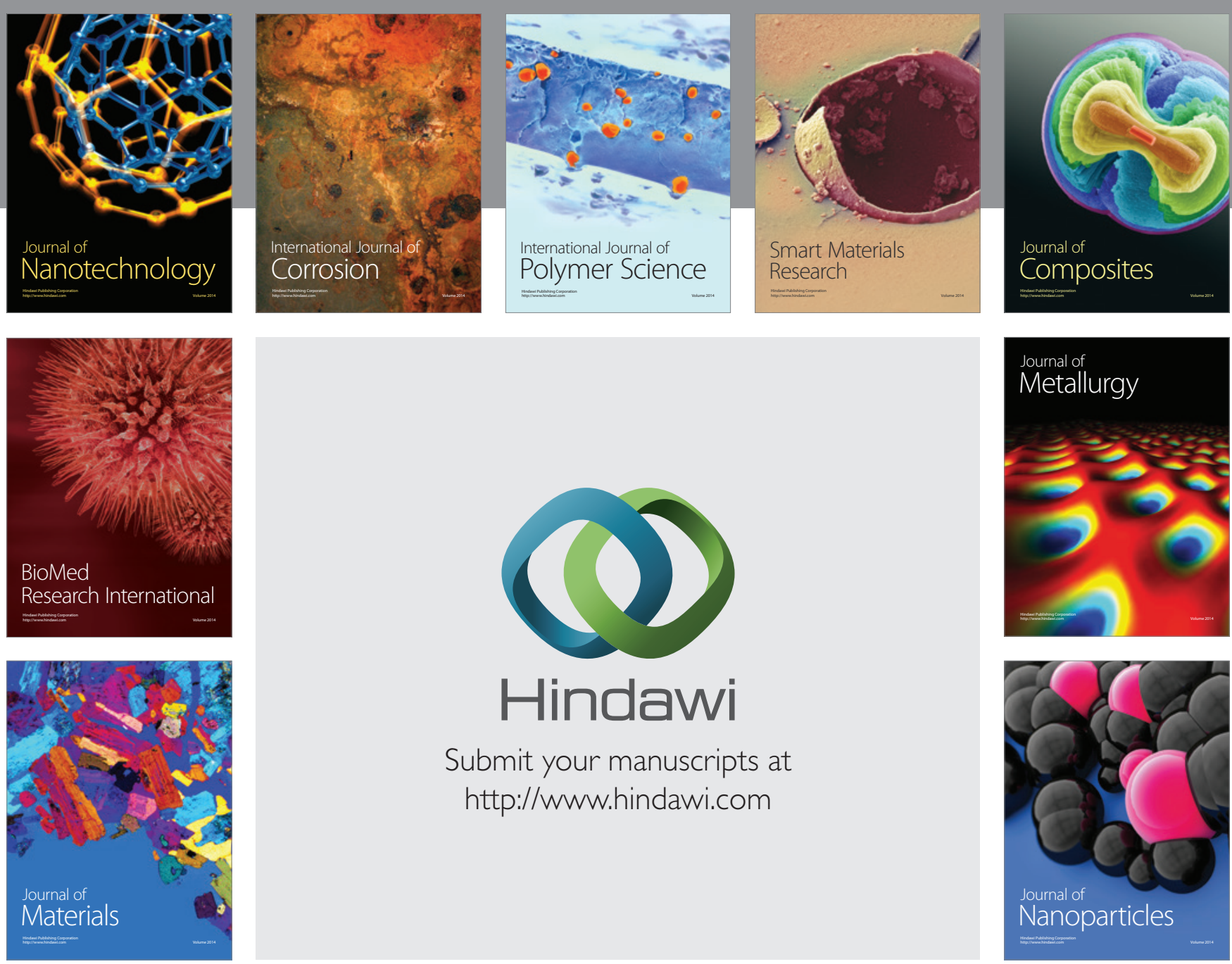

Submit your manuscripts at http://www.hindawi.com
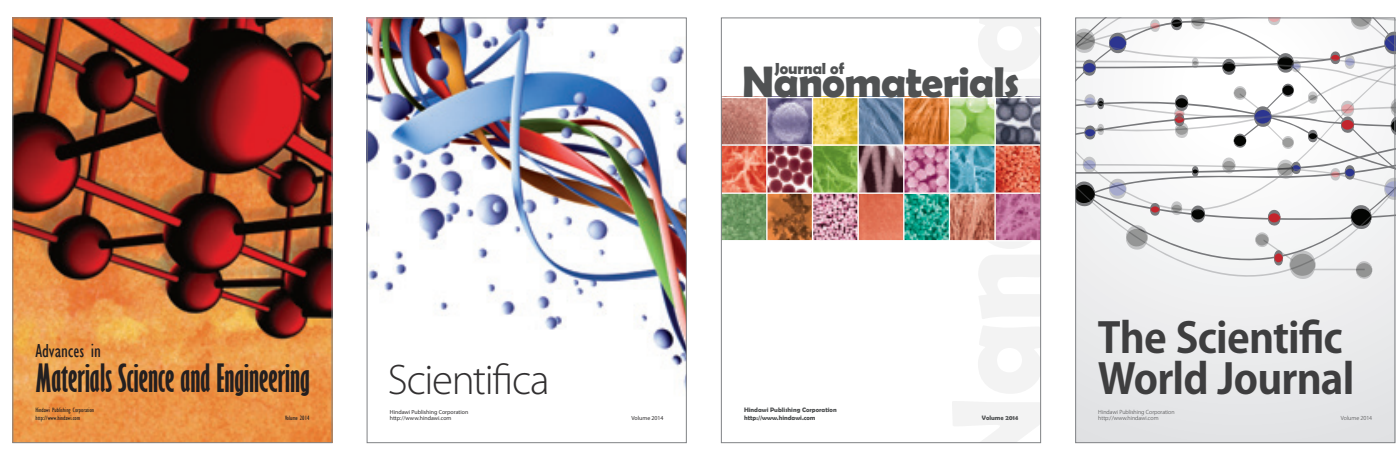

\section{The Scientific World Journal}
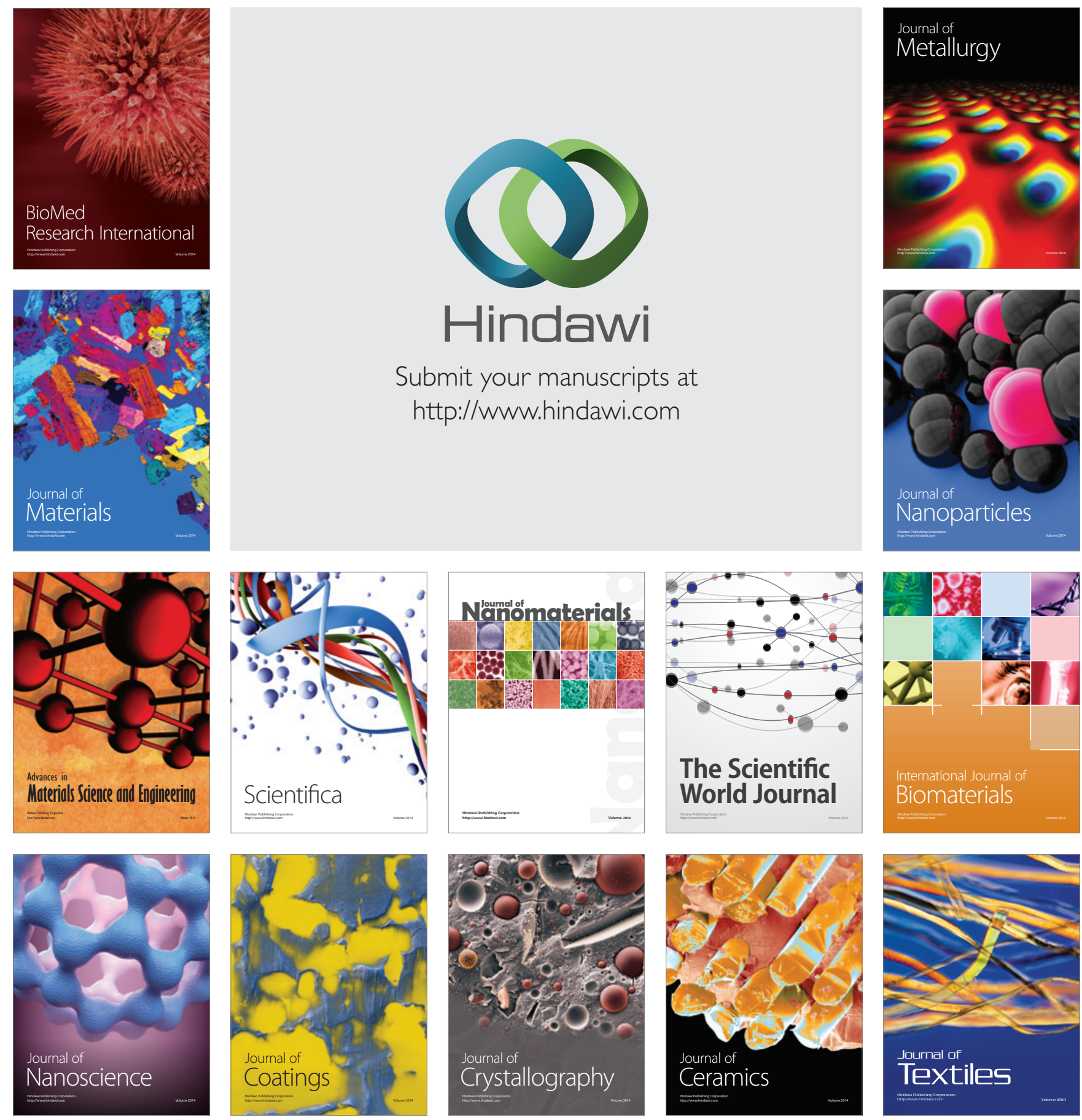